\title{
Cold expansion process on hard alloy holes-experimental and numerical evaluation
}

\author{
Victor Achard ${ }^{1,2, a}$, Alain Daidie ${ }^{1}$, Manuel Paredes ${ }^{1}$ and Clément Chirol ${ }^{2}$ \\ ${ }^{1}$ Université de Toulouse, INSA/ICA (Institut Clément Ader), 135 avenue de Rangueil, 31077 Toulouse Cedex 04, France \\ 2 Airbus Operations S.A.S., 316 route de Bayonne, 31060 Toulouse Cedex 09, France
}

Received 2 December 2014, Accepted 27 September 2015

\begin{abstract}
This paper presents an evaluation of the influence of the cold expansion process on the fatigue performance of holes in hard alloys, as such materials are involved in an increasing number of aeronautical applications. Fatigue enhancement could bring significant savings for numerous possible industrial applications. However, although the cold expansion of aluminium holes has been studied widely, there have been few publications concerning this process in hard alloys and there is currently very few research activities on technique dedicated to high strength metallic holes. Thus, this work aims to define an approach to understand why processes and methodologies are suitable for obtaining effective expansion of these materials. In this article, the response of expanded holes is studied, considering various experimental parameters and for a wide range of expansion ratios. The influence on the fatigue strength of Ti-6Al-4V tensile specimens is also reported for various expansion ratios, more precisely "standard" expansion ratios and high expansion ratios. Then, a specific numerical modelling of the process is presented, which enables us to understand the impact of high expansion ratios in titanium holes and the influence on fatigue performance. A very good correlation between experimental and numerical results is observed.
\end{abstract}

Key words: Cold expansion process / hard alloy / fatigue performance / expansion ratio / axisymmetric F.E.M.

\section{Nomenclature}

\begin{tabular}{|ll|}
\hline$D$ & Diameter of the hole $\left(10^{-3} \mathrm{~m}\right)$ \\
$I_{e}$ & Initial expansion ratio $(\%)$ \\
$L$ & Length of the specimen $\left(10^{-3} \mathrm{~m}\right)$ \\
$R_{e}$ & Residual expansion ratio $(\%)$ \\
$T$ & Thickness of the specimen $\left(10^{-3} \mathrm{~m}\right)$ \\
$W$ & Width of the specimen $\left(10^{-3} \mathrm{~m}\right)$ \\
\hline
\end{tabular}

\section{Introduction}

\subsection{Optimizing the use of hard metals in aircraft structures}

In the field of aeronautics, further understanding of the behaviour of materials under complex stress fields has become a serious challenge. It can lead to the optimization of fatigue performance of parts and assemblies which, in turn, can work towards a highly desirable weight reduction of the structure and thus increase the efficiency of aircraft. This search for optimization is currently expressed

\footnotetext{
${ }^{a}$ Corresponding author: victor.achard@airbus.com
}

in the increasing use of high-performance materials, particularly in the primary structure. These new materials include, in particular, the family of hard alloys, whose physical properties often allow the best compromise to be found between density and the characteristics needed for the application. The hard alloy family comprises metallic alloys with ultimate tensile strengths close to or higher than $1000 \mathrm{MPa}$ at $20^{\circ} \mathrm{C}$. Regarding the different justifications for the use of hard metals in the design of an aircraft structure, optimization of the various components is a major requirement. On the other hand, in modern aircraft, these materials enable the integration of a large proportion of composite materials (CFRPs) as structural material. They ensure good galvanic and thermal dilation compatibility in multi-material hybrid connections together with good shock response of the overall structure. Finally, new engines, running at ever higher speeds and temperatures, open up many possibilities for applications where hard metal fatigue becomes a significant problem. Ezugwu et al. [1] recall that each kilogram removed from the total mass of a jet engine saves 150000 USD of fuel over the engine's working life. Despite all the advantages offered by this family of metals, the costs they generate make it necessary to optimize their use, by adapting 


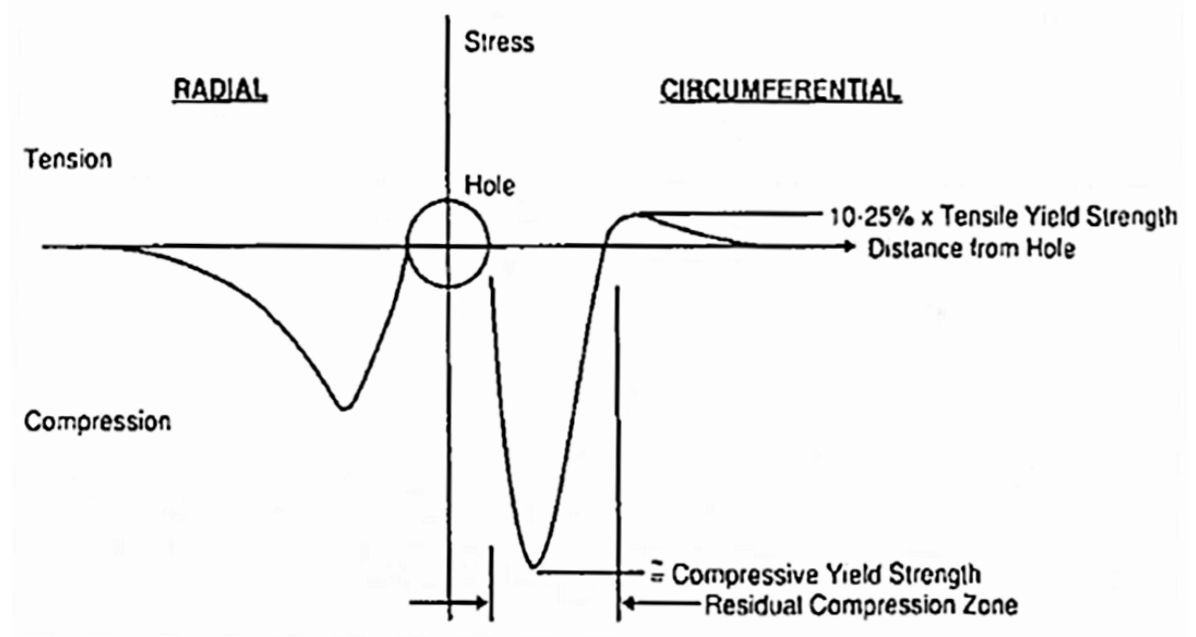

Fig. 1. Biaxial stress field in the hole edge after expansion [9].

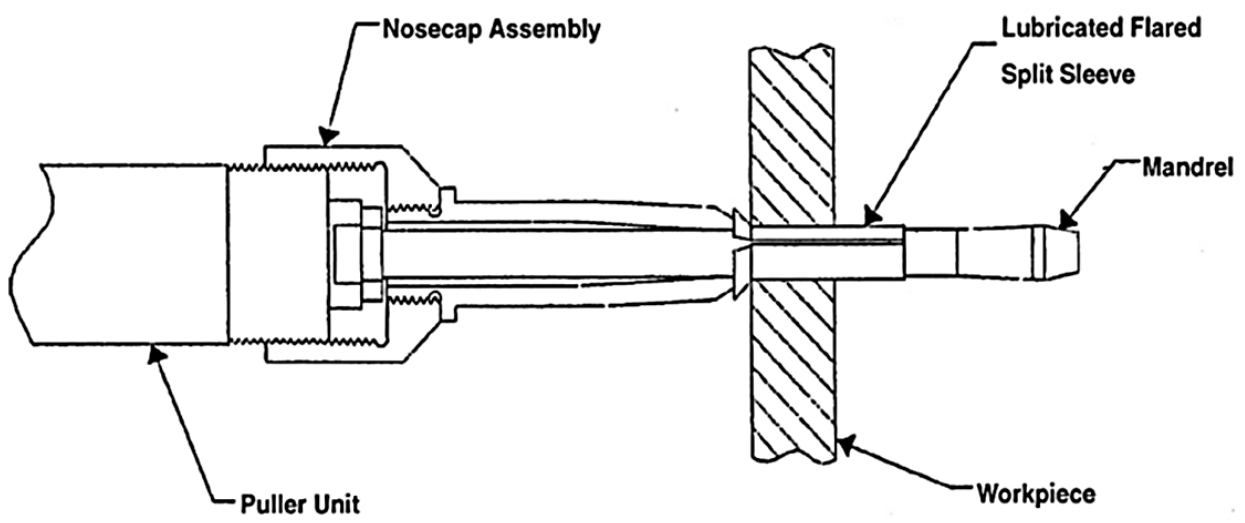

Fig. 2. Split sleeve cold expansion process, from [29].

their morphologies and therefore their characteristics according to their loading environment, especially in the aim of resisting fatigue. Moreover, in areas prone to fatigue issues, few industrial processes exist for service life extension of hard metals parts. Thus, there is a real need for a cheap and efficient maintenance (or repair) solution for these components, in order to prevent expensive but necessary part replacement or re-design. As these alloys are often used in areas of aircraft where accessibility is poor, the importance of such issues is even more capital.

\subsection{Improvement of fatigue performance using cold expansion process}

This study focuses on optimizing the fatigue performance of bolted joints using hole cold expansion. Aeronautic structures consist of a large quantity of riveted or bolted sub-assemblies that require the presence of fastening holes. These sections are often critical in the design and act as major sources of stress concentration where fatigue damage starts [2]. Thus, it has been observed that, statistically, fatigue of bolted joints accounts for 50-90\% of all of the cracks sustained by an aircraft in operation [3]. Hole cold expansion involves the generation of compressive residual stress fields induced by hardening and modifies the kinetics and onset areas of the initial cracks, which will later spread to a less critical region beneath the surface of the material [4]. The material is thus subjected to compressive and tensile residual stresses that are in overall equilibrium, the compressive stresses generally being balanced by a tensile field away from the hole [5]. In order to be optimal, radial expansion of the hole should induce circumferential stresses close to the compressive limit at which the material yields. They thus compensate for the mean value of future tensile fatigue loading (Fig. 1). This process reduces the criticality of these joints as methods involving the control of the fastener preloads [6] or interference fit [7].

Among the existing processes designed to generate extensive and controllable residual stress fields at the edge of the hole while minimizing damage to the hole surface, split sleeve cold expansion (Fig. 2) remains the most widely used in industry $[8,9]$. 
From a technological point of view, a split sleeve is inserted in an initial hole in order to protect it and the cold working is performed by pulling an oversized tapered mandrel through the assembly. Then, the cold expanded hole is reamed to its functional diameter. This process greatly improves the fatigue performance of the hole. For example, it is commonly noted that the lifespan of a hole that has been cold expanded using the split sleeve process is three to ten times that of an "as drilled" hole in aluminium alloys such as 2024 T351 [10,11]. In other words, gains on the fatigue quality index can reach $+30 \%$. Because of the simplicity of the operations involved and the gains observed, this process is very efficient in improving the quality, reliability and performance of bolted joints during their design, repair or post-production phases. Thus, it appears interesting to evaluate the cold expansion process on hard metals by observing their responses and the potential fatigue gains that could be generated. In the literature, few studies describe the application of the cold expansion processes to hard metal and, because of the very specific mechanical and metallurgical characteristics of these materials, it seems impossible to make a direct analogy with other materials.

\subsection{Hole cold-expansion process for hard alloy applications}

The first studies, dating from the 1970s, by Philips [12], Rich and Impellizzeri [13] and Sha et al. [14] report that the cold expansion process was applied to hard alloys and concerned almost exclusively titanium alloys. Some interesting results can be found in the literature, obtained on tensile specimens in "open-hole" or "filledhole" configurations having width-to-diameter ratios between 4 and 5. Philips [12] performed tests on hard metals such as Ti-6Al-4V, Ti-6Al-6V-2Sn, or AISI4340 steel. He obtained gains in fatigue performance on numerous configurations, even though the number of specimens tested was small. More precisely, fourfold gains in lifespan of the specimens $(+23 \%$ on the fatigue quality index $)$ were observed on Ti-6Al-4V specimens with a split sleeve expansion of $5 \%$ on a $9.52 \mathrm{~mm}$ hole. In the same way, Rich and Impellizzeri [13] observed fourfold increases in the lifespan of $6.35 \mathrm{~mm}$ "filled-hole" Ti-6Al-4V specimens (i.e. specimens containing a pin with clearance fit), which were expanded (4\%) with the split sleeve process.

During the same period, a publication by Rufin [9] reported interesting results obtained after expansion of isolated hard metal applications, almost exclusively titanium alloys, with or without bush installation. These studies were mainly performed on behalf of industry or military organizations. Testing concerned very specific areas: engine parts, clevises or other specimens and sections involving high load transfer. The test cases selected were representative of applications where the expansion appeared to be a solution for repairing the structural detail or improving its fatigue performance. However, over this period, no precise analysis was made of the response of hard metals subjected to expansion and only studies of specific cases can be found, whereas generic processes designed for the expansion of holes in aluminium alloys were considered. In addition, it is difficult to quantify the fatigue gains obtained in these studies as the various configurations tested involved many parameters influencing fatigue behaviour. Furthermore, scatter on the results is large and reduces the validity of the assumptions made. More recently, two general studies have been conducted by Yan et al. [15] and Liu et al. [16] regarding Ti-6Al$4 \mathrm{~V}$ hole expansion. In addition, they observe its influence on crack propagation rate. Again, the split sleeve process is considered and threefold increases are observed in the lifespan of cold expanded (4\%) $6.35 \mathrm{~mm}$ "open-hole" Ti-6Al-4V specimens [15].

Finally, a process where residual stresses are set up by indentation of the surfaces has also been tested $[17,18]$ on tensile specimens and medical implants made of Ti-6Al$4 \mathrm{~V}$. The authors observed that the lifespan of the tensile specimens was multiplied by 4.7 .

So studies concerning the expansion of hard metals mainly come down to extensions and implementations of processes and methodologies already used on aluminium alloys. In addition, the influence of the thickness and the diameter of the hole is not considered, neither is the influence of the expansion ratio. Thus, there are few quantitative results available on the response of expanded hard metal holes and there has been no research concerning dedicated processes for expanding hard metal holes. Their highly specific behaviour, concerning both thermal and metallurgical issues or regarding the integrity of the surfaces generated, are not taken into account for the installation of residual stresses at the hole edge. Finally, these studies concern almost exclusively Ti-6Al-4V applications. Thus, it appears interesting to generalize cold expansion to holes in the hard alloys that are the most widely used within aircraft structures nowadays, such as precipitation hardening stainless steels and nickel-based alloys.

\subsection{Numerical modelling of the cold expansion process in hard alloys}

The main difficulties in studying the expansion process in hard metals arise, first, from the behaviour of these materials when subjected to expansion. Their static and fatigue response appears very different from that observed on aluminium alloys. Also, this family of materials exhibits significant disparities concerning specific strength (stress-strain up to failure) and monotonic behaviour. For example, the mechanical properties of titanium alloys are highly dependent on their thermo-mechanical loading history and initial composition and microstructure. Thus, alloys such as Ti-6Al-4V in annealed condition predominantly contain $\alpha$ phase $(\mathrm{HCP})$ at ambient temperature and exhibit typical anisotropic mechanical properties, particularly tension-compression asymmetry. Moreover, this behaviour is highly dependent on strain ratio and temperature [19-22]. Therefore, this material commonly reveals compressive failure stresses and strains 
that are much greater than their tensile counterparts. On the other hand, the materials may exhibit significant kinematic behaviour, which appears particularly important in titanium alloys, for example. This behaviour is attributed to the limited number of slip systems in hexagonal closepacked ( $\alpha$-phase) material at ambient temperature [23]. Thus precise characterization of the materials considered is important in order to understand the various phenomena observed and to be able to simulate them.

Numerical methods such as finite element modelling appear to be efficient tools for predicting the response of the material and particularly the residual fields generated, on condition that the modelling strategies are sound. There are numerous studies concerning the split sleeve expansion process in the literature $[24,25]$. However, in the case of hard alloys, we found only two $[9,15]$. The modelling choices must be clearly selected in order to simulate highly non-linear behaviours such as high plastic flows and high strain rates. These issues are especially justified by the localized phenomenon caused by the split of the sleeve, the complexity of the intrinsic behaviours of hard metals, the various contacts affecting the material and the nature of the tools used for the expansion. Because of the one-sidedness of the application of the expansion, major axial loads are generated and cause significant side effects. For all these reasons, it is well known that residual fields in the depth of the hole are highly non-uniform in the case of the split sleeve process. More precisely, a decrease of the residual circumferential stresses at the entrance side of the mandrel is commonly observed. These areas are poorly expanded and reduce the efficiency of the process, the least compressive areas being the most vulnerable. While considering hard alloys, Liu et al. [16] numerically identified relatively low circumferential residual stress in the entry side of the mandrel, of about $-300 \mathrm{MPa}$ and therefore far from the compressive yield stress of Ti-6Al-4V. This phenomenon was also observed by Rufin [9]. In order to overcome this problem, various experimental methods have been developed, from SWCW (Stress Wave Cold Working) patented by the Stress Wave Company [26] to the process designed by Chakherlou et al. [27], which generates nearly uniform residual fields.

The modelling choices generally associated with the expansion processes are the use of a 3D model with an isotropic or kinematic elasto-plastic constitutive material law and a Von Mises yield criterion. This strategy is especially justified by the localized phenomena caused by the presence of the split of the sleeve on the hole during expansion. However, some simplified 2D models have been developed. Actually, it has been shown that the residual stress field is no longer disturbed by the presence of the split when we move slightly away from its zone of influence along the circumference [15]. In our problem, axisymmetric modelling appears sufficiently representative of phenomena operating over the vast majority of expanded sections, at least those that will be the most loaded during subsequent use. Moreover, that kind of modelling strategy allows the dependence of simulations on various param- eters, such as the material law, the various coefficients involved and the geometries and expansion ratios, to be quickly assessed.

\subsection{Methodology}

In the present work, the first step is to evaluate experimentally the feasibility of the split sleeve expansion process in hard alloy holes. These tests are performed in order to highlight the specificities and response to the expansion in various typical hard alloys, testing various configurations and ranges of expansion ratios. This first characterization was performed on specimens made of titanium alloy ( $\alpha \beta$ annealed Ti-6Al-4V), martensitic stainless steel (precipitation hardening) and nickel-based alloy (nickel chromium corrosion-resistant superalloy). The chemical compositions of these materials are given in the aeronautical standard. These tests aim mainly to determine the physical limits of the process, ensuring that the operating characteristics of the hole are maintained. Afterwards, we analyse the influence of expansion on the fatigue performance of elementary tensile specimens. This step is harder to implement, in term of tests conducted and machining of the specimens. Thus, in this study we focused only on the Ti-6Al-4V, this alloy being indeed the most used in the aeronautical field and where the potential of industrial application for the cold expansion is much more important. Wohler curves of open-hole specimens are studied, considering not only "standard" expansion ratios (i.e. similar to those used on aluminium alloys) but also high expansion ratios. Two hole diameters, commonly used in aeronautic assemblies, are studied. Then, we analyse the results achieved via axisymmetric FE modelling of the split sleeve process. The numerical work concerns the study of the expansion of the Ti-6Al-4V and is intended to explain some of the results observed during the previous fatigue campaign. Values and distribution of circumferential stresses provided by the simulations allow the efficiency of the process to be estimated considering different expansion ratios. A parametric study regarding the hardening law taken is presented and results are compared and correlated to experimental values in order to determine what modelling strategy is most suitable. Thus, these results provide an understanding of whether the processes designed for expansion in aluminium alloys are suitable in terms of expansion ratios, and determine what physical phenomena are involved. More generally, these first results provide information regarding the application of the expansion process to hard alloys and define an approach for understanding which processes and methodologies are suitable for the efficient expansion of hard metals.

\section{Experimental procedure: influence of cold expansion process in hard alloy holes}

\subsection{Split sleeve expansion of hard alloy holes}

This experimental study aimed to evaluate the behaviour of hard alloy specimens when they were subjected 


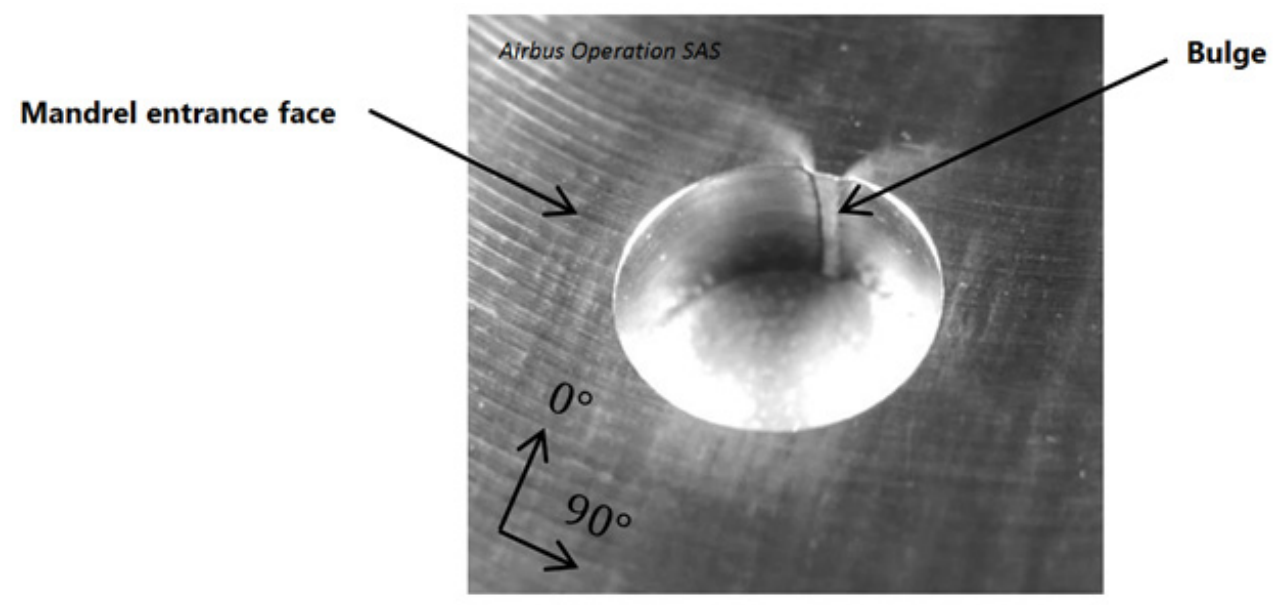

Fig. 3. A cold expanded Ti-6Al-4V $9.52 \mathrm{~mm}$ hole - View of the affected zone.

to expansion, to observe whether a plastic strain field might be generated in the hole edge and if fatigue gains were indeed observed. The aim was thus to see if expansion was achieved easily in hard metals and to evaluate potential associated risks, such as the generation of cracks at the hole edge. From the technological point of view, the split sleeve expansion process was chosen. This process can, basically, be divided into six key steps. First of all, after dimensional control of the original hole, a prelubricated split sleeve is placed on the mandrel, which is connected to the hydraulic actuator. The split sleeve is slid along the maximum diameter of the mandrel and positioned on the minimum diameter so that the tooling can be inserted from one side of the hole. The angular position of the split is then carefully adjusted to ensure that the affected zone (extensive distortion) will not correspond to the main directions of loads operating on the treated part. In Figure 3, the bulge caused by the split sleeve is clearly identified. Moreover, this bulge is wider on the top face, which corresponds to the entrance face of the mandrel. The actuator is subsequently triggered and the mandrel is pulled through the sleeve and the hole, performing the expansion. In the split sleeve process, the sleeve is then removed from its housing. Here, the prelubricated sleeve is useful to reduce the axial effort involved in pulling the mandrel and to protect the contact surface. Finally, the hole is reamed to its functional diameter, and checked. It is then ready to receive a fastener, for example. The theoretical expansion ratio applied $\left(I_{e}\right)$ is defined by relation (1). Here, a wide range of expansion ratios is to be tested, high ratios being obtained by reducing the initial hole diameter while keeping the tooling diameter constant.

$$
I_{e}=\frac{\emptyset \text { tooling }-\emptyset \text { initial }}{\emptyset \text { initial }}
$$

where the tooling diameter corresponds to the maximum diameter of the mandrel and sleeve assembly, and the initial diameter is the diameter measured after initial drilling of the specimens.
Here, holes were machined with orbital drilling in order to reduce residual stresses generated on the surface of the hole and reduce the scatter of results during the tests. Similarly, Ti-6Al-4V specimens were heat treated to relieve stress after milling of the external faces. Maximal tolerance on the hole diameter was $\pm 0.02 \mathrm{~mm}$, which ensured good accuracy of the expansion ratio actually applied. Expansion was first performed on "5 hole" feasibility specimens, always in the plane of the sheets (longitudinal-longitudinal/transverse orientation). These normalized specimens had a width equal to 3 times the nominal diameter (free-edge distance) and the distance between 2 holes was equal to 5 nominal diameters to avoid reciprocal influence. Various expansion ratios were tested on two main diameters commonly used in aeronautic assemblies (6.35 $\mathrm{mm}$ and $9.52 \mathrm{~mm}$ ) and the thickness of the specimens was $5 \mathrm{~mm}$. Two orientations of the sleeve relative to the main direction of grain flow were considered $\left(0^{\circ}\right.$ or $\left.90^{\circ}\right)$, the fracture toughness of the material being particularly different in these two directions. A specific mandrel was designed and manufactured which, because of its geometry and its material, allowed high expansion ratios to be reached without excessive distortion or wear of the tapered section.

During these tests, no cracking of the material was observed in the microscopic inspection and Rototest nondestructive testing (Fig. 4). The residual expansion ratio was analysed with respect to the theoretical applied ratio. The residual expansion ratio was obtained by measuring the cold worked hole (residual hole) using an internal micrometer (out of the affected zone). The residual expansion ratio $\left(R_{e}\right)$ was calculated for each specimen and defined by relation (2).

$$
R_{e}=\frac{\emptyset \text { residual }-\emptyset \text { initial }}{\emptyset \text { initial }}
$$

Figure 5 brings data collected during the feasibility test campaign. More precisely, it concerns the analysis of the residual expansion ratio. The spread of results is represented for the worst case measured for each material. 

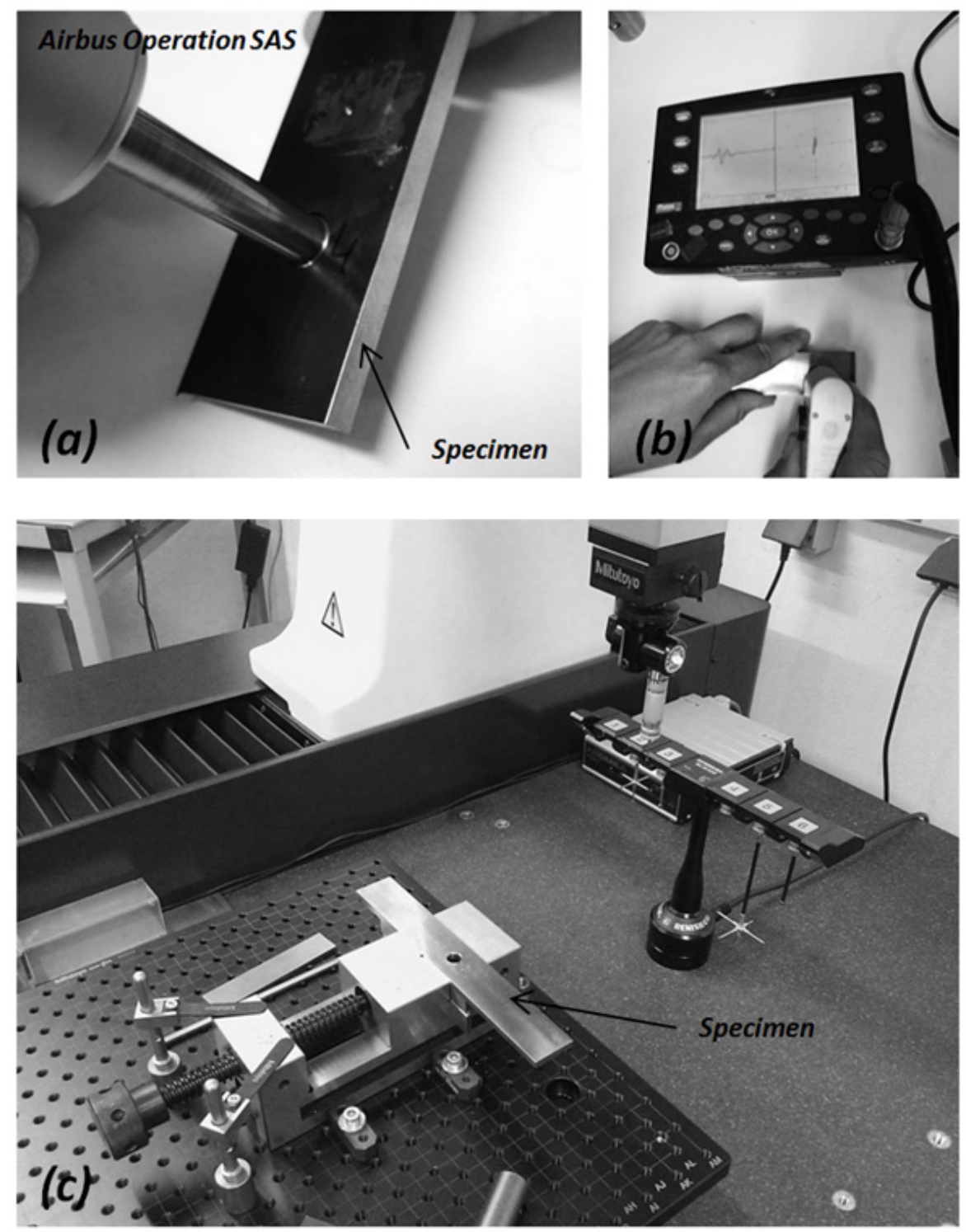

Fig. 4. Rototest control of a cold expanded hole (a), Rototest device (b), 3D Measurement machine (c).

First of all, it can be noted that the plot of the residual expansion according to the initial expansion ratio serves to highlight the characteristic behaviour of these metals. These residual expansion ratios were obtained from different expansion levels, and each point corresponds to the mean value obtained on 5 holes at least. These curves are plotted only for $5 \mathrm{~mm}$ thick specimens. The nearly perfect linear correlation between these two values is significant, given that the parameters considered here during the process (materials, configurations, contacts) are almost exclusively non-linear. Furthermore, the spread of results appears very low in these tests, indicating very good reproducibility of the process. This low spread can be explained in particular by the precise machining of the specimens and the tooling, and also by the fact that the batches of material had the same origins and the rolling orientations were controlled. Regarding the titanium alloy Ti-6Al-4V, highly linear behaviour can be seen when the expansion ratio increases. In addition, the slope and constant terms of the curves are relatively low (lower than for aluminium), a phenomenon explained in particular by the low modulus (high springback) and high strength of the material. On the other hand, the behaviour of the nickelbased alloy appears very different from that of a titanium alloy or a stainless steel, its residual expansion being significantly higher than for other materials. In comparison, for "standard" expansion ratios (3\% and 4\%), 2024 and 7010 aluminium alloys exhibit residual expansion ratio between those of the steel and the nickel-based alloy. It can thus be argued that it is necessary to impose very high expansion ratios in titanium and steel to achieve a sufficient level of plasticity and counterbalance elastic feedback of the hole.

This graph also shows the independence of the expansion behaviour with respect to the hole diameter. The experimental curves obtained for $9.52 \mathrm{~mm}$ and $6.35 \mathrm{~mm}$ 


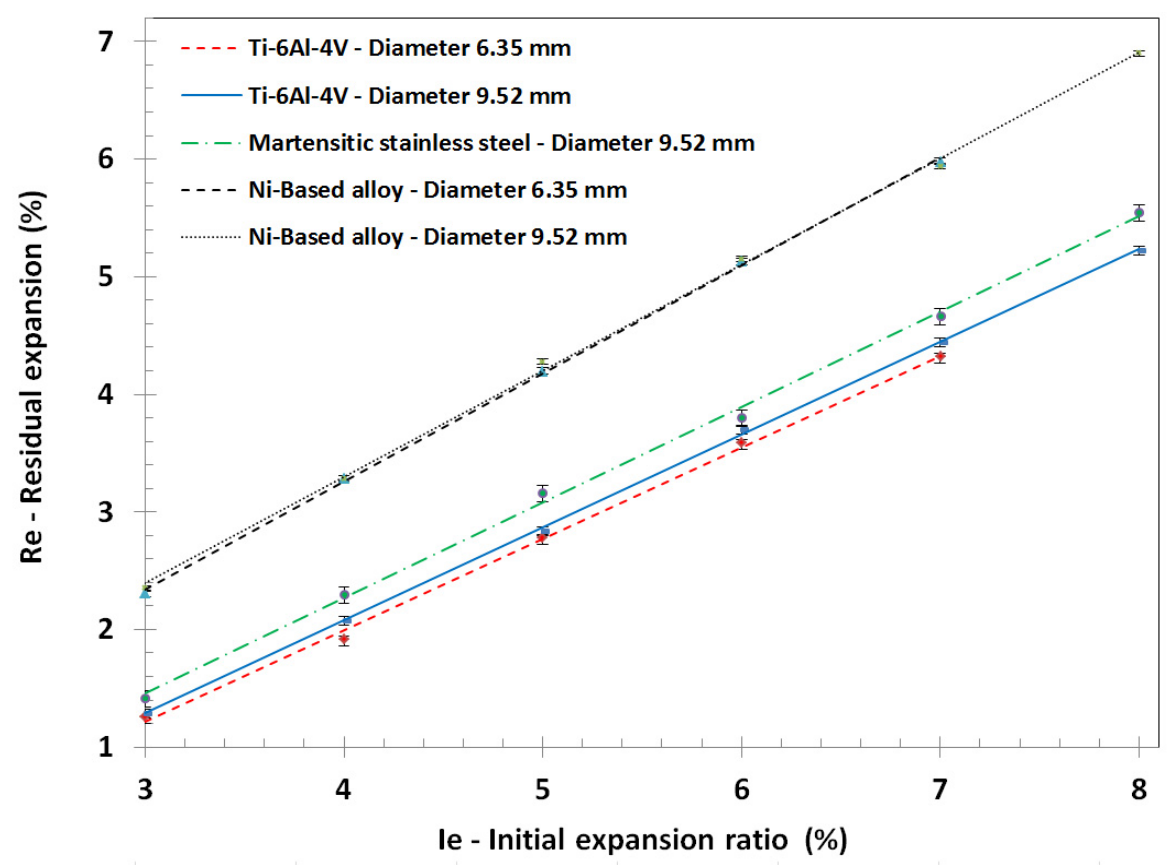

Fig. 5. Evolution of the residual expansion in hard alloy holes.

diameter holes are extremely close (titanium and nickel based alloy). This result is very interesting and allows the absence of scale effect to be assumed in the first instance. These results indicate that the split sleeve process can be successfully applied to hard alloy holes. Moreover, it appears that these metals are able to sustain high expansion ratios and that there is no apparent saturation of the behaviour of the materials. Results suggest that the hardening continues at the edge of the hole when expansion ratio increases. The influence of these high expansion ratios on the fatigue performance of the hole remains to be seen, so the next section discusses the influence of high expansion ratio on the fatigue strength. This is indicative of the performance improvement of the area and therefore allows the efficiency and effectiveness of the process to be objectively quantified.

\subsection{Fatigue performance of $\mathrm{Ti}-6 \mathrm{Al}-4 \mathrm{~V}$ specimens}

After noting that cold expansion using the split sleeve process was functional on hard alloy holes, we sought to observe the fatigue behaviour of expanded Ti-6Al-4V specimens. After observation of the non-influence of the hole diameter during feasibility tests, it was interesting to check whether fatigue followed the same trends. Thus we tested conventional "standard" ratios (i.e. those applied during expansion for aluminium) and higher ratios, which were expected to be well tolerated by the material. "Open-hole" T-Type elementary fatigue specimens were manufactured and expanded. The objective was to assess the influence of the cold expansion and also the influence of the expansion ratio. The specimens tested in fatigue were $5 \mathrm{~mm}$ thick and drilled to their initial expansion diameter with a maximum tolerance of $\pm 0.02 \mathrm{~mm}$. The

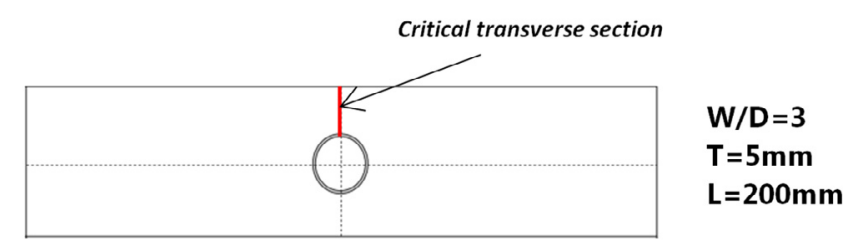

Fig. 6. Overall geometry of fatigue specimens (all dimensions in $\mathrm{mm})$.

general geometry of the fatigue specimens is presented in Figure 6 . These normalized specimens had a width equal to 3 times the nominal diameter, $6.35 \mathrm{~mm}$ and $9.52 \mathrm{~mm}$ in our case. The complete length of the specimens was $200 \mathrm{~mm}$. They exhibited a stress intensity factor close to 2.3 in the net section (gross section minus hole area), ensuring that the hole edge would be the place of onset of cracking and thus the weak point of the specimens.

The overall average surface roughness of a specimen (hole and outer surfaces) was less than $1.6 \mu \mathrm{m}$ to avoid potential locations of crack onset in other places. In order to decrease the scatter on test results, specimens were first treated to relieve stress and holes were then machined by orbital drilling. Specimens were first expanded to the theoretical ratio, then reamed (orbitally) and chamfered to their nominal diameters. Again, maximum tolerance on hole diameters was $\pm 0.02 \mathrm{~mm}$ (Fig. 7).

Fatigue tests were performed by using a sinusoidal wave of a tensile-tensile load spectrum $(R=0.1)$, with a frequency of $10 \mathrm{~Hz}$ and for various stress levels, to obtain Wohler curves. These stress levels correspond to the average theoretical longitudinal stress in the net section of the specimen (gross section minus hole area) that is reached for the maximum load applied during a fatigue cycle. The 


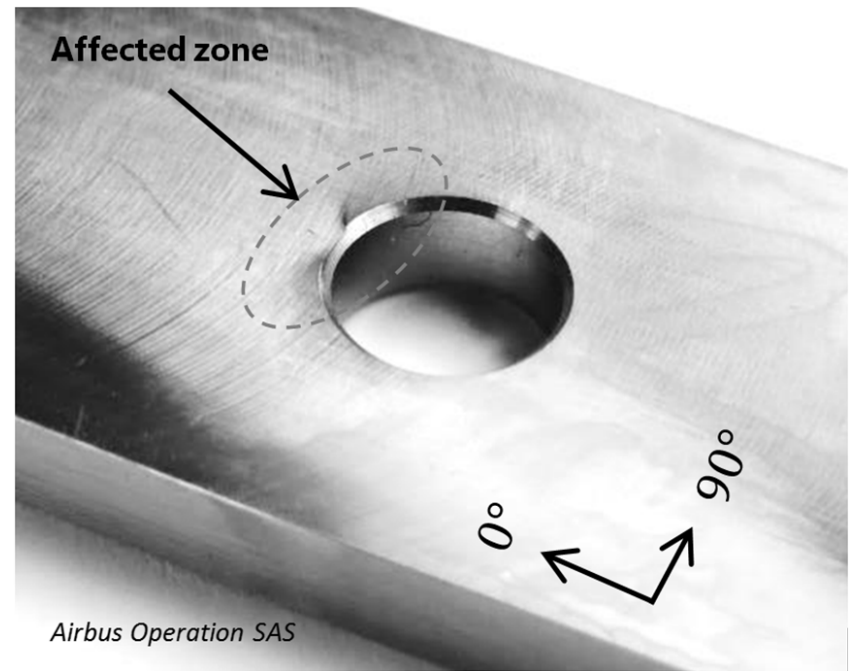

Fig. 7. A cold expanded Ti-6Al-4V $6.35 \mathrm{~mm}$ hole after reaming.

curves (Fig. 8) show the logarithmic trend lines of Wohler curves plotted for baseline specimens "as drilled" and for 2 different expansion ratios, each curve being obtained from the test results of 8 specimens. These curves are plotted with $50 \%$ reliability on the number of cycles sustained until failure of the specimens (as many specimens on the left as on the right of the curve). In the aeronautical industry, the fatigue performance of a structure is often evaluated by determination of its fatigue quality index, which corresponds to the stress associated with a forecast fatigue life of $10^{5}$ cycles. In the following graphs (Fig. 8), the stress levels are normalized by the fatigue quality index of the baseline (without expansion). Normalization allows to quickly see the benefits in terms of stress that can be sustained by the structure.

The fatigue quality index is computed using industrial software that generates an interpolation Wohler law (50\% reliability) from the experimental results. We can see that the improvement in fatigue performance is verified for "standard" expansion ratios and is enhanced with higher ratios. These results were verified on both $6.35 \mathrm{~mm}$ and $9.52 \mathrm{~mm}$ holes. For $6.35 \mathrm{~mm}$ holes, the fatigue quality index was $17 \%$ higher using the "standard" expansion ratio and $27 \%$ higher using high expansion ratios. For $9.52 \mathrm{~mm}$ holes, the fatigue quality index was $15 \%$ higher using "standard" expansion ratio and 30\% higher using high expansion ratios. The fatigue life ratios were close to $3: 1$ at least for the "standard" ratio and to $6: 1$ for high ratios. Improvements were in the range of those found in the literature review (Sect. 1.2).

Moreover, we can observe that the influence of the high expansion ratios is even more notable for the low number of loading cycles ( $<200000$ cycles). Thus, the gains in terms of failure stress are doubled in this range of cycles compared with lower expansions ratios. This aspect is particularly interesting because the aircraft components are usually designed for this range of load cycles.
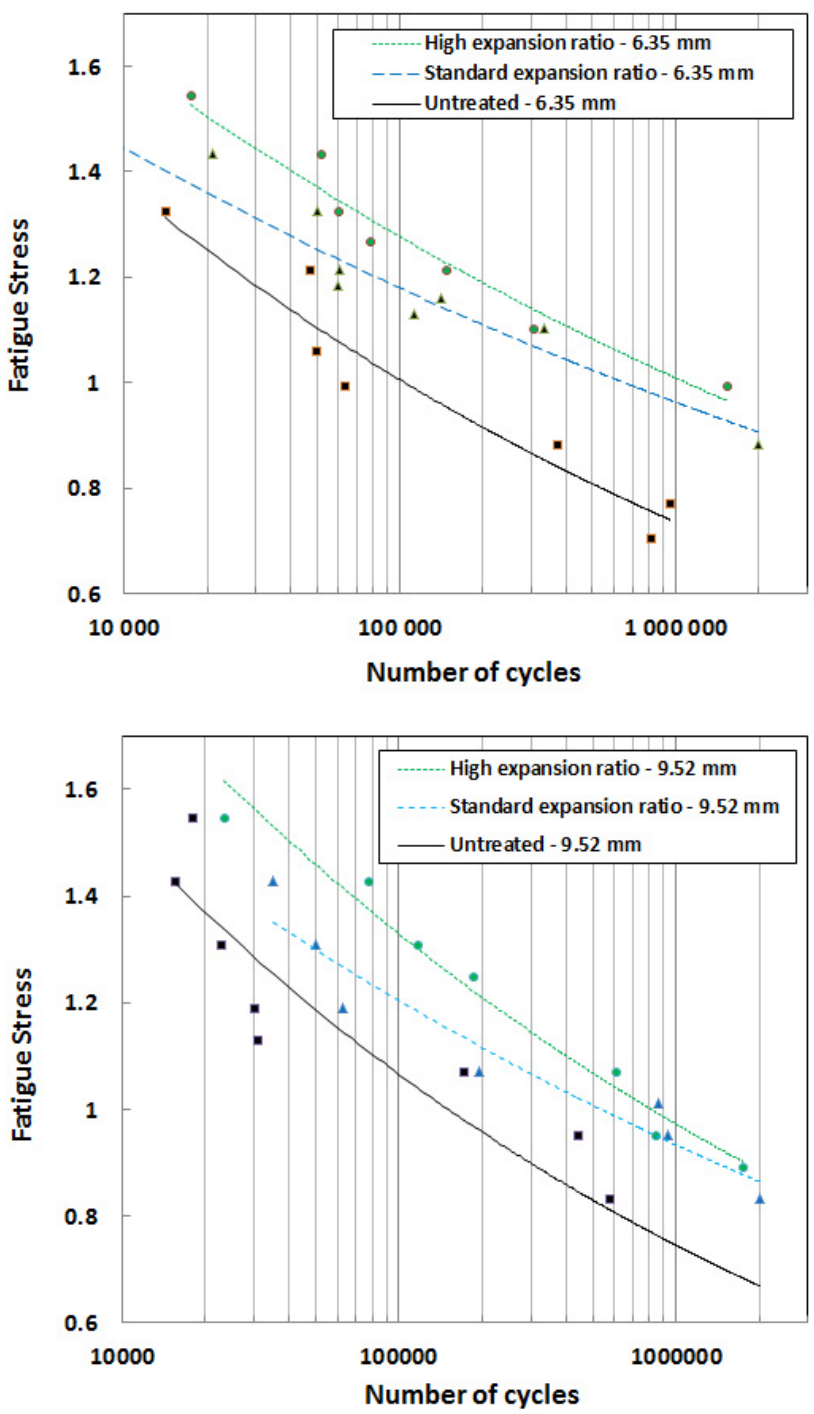

Fig. 8. Fatigue tests of Ti-6Al-4V expanded "open-hole" specimens.

On the other hand, we can see that for both expansion ratios, gains on fatigue limits are similar.

Regarding the failure modes (Fig. 9), large majority of specimens failed in the net section (i.e. the lower section that contains the hole). Crack initiation occurs from the edges of the hole and propagates in the net section. Beyond this propagation region, we notice static failure (Fig. 9a). This failure mode is observed for both expanded and non-expanded specimens. On the other hand, another failure mode may be observed for high expansion ratios and for high numbers of cycles (>300000 cycles). It is characterized by an initiation that occurs deep below the surface, with an offset distance from the original critical cracking plane (Fig. 9b). This failure mode indicates the change in position of the maximal stress concentration, initially located in the hole edge. Generalized failure of the coupons then occurs on the side or not of the defect generated by the split sleeve (Fig. 9c). 


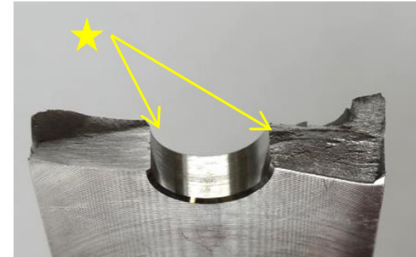

(a)

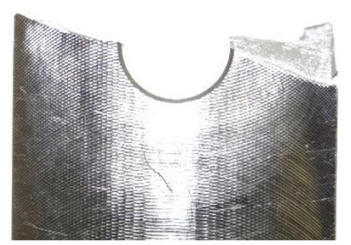

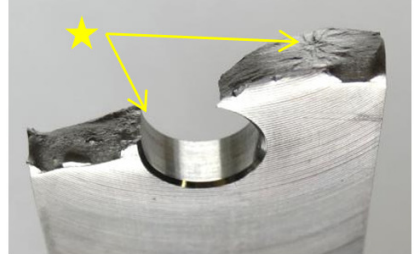

Split defect

(b)

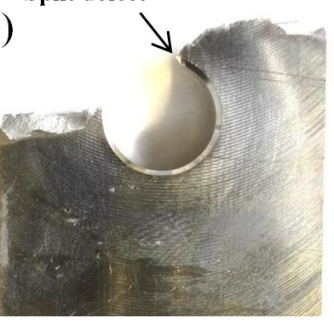

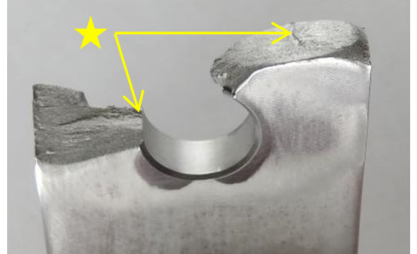

(c)

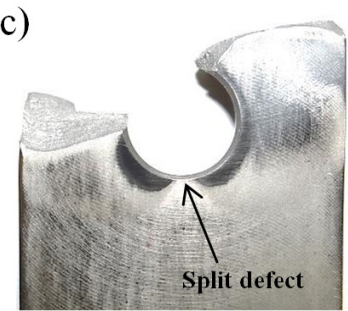

Fig. 9. Overview of the failure patterns observed after fatigue of cold expanded $9.52 \mathrm{~mm}$ Ti-6Al-4V T-type specimens: netsection failure (a), "offset" failure, split sleeve at $0^{\circ}$ (b), "offset" failure, split sleeve at $180^{\circ}$ (c).

In order to understand these improvements in fatigue performance and the influence of the use of a higher expansion ratio, a numerical model was developed to determine the response of $\mathrm{Ti}-6 \mathrm{Al}-4 \mathrm{~V}$ subjected to several expansion ratios.

\section{Axisymmetric FE modelling of the split sleeve expansion process in hard alloys}

\subsection{Model definition}

This section reports the results obtained through FE modelling of the split sleeve process in hard alloy holes and, more precisely, in the case of a Ti-6Al-4V in $\alpha \beta$ annealed condition. Axisymmetric modelling was chosen for the reasons given in Section 1.3, in order to obtain a fast, polyvalent model that allowed numerous geometries and expansion ratios to be considered and, later, various materials. The model presented in Figure 10 was established using the commercial program ABAQUS 6.12 and the results were obtained using the ABAQUS Standard implicit solver.

The geometries, loading environment and boundary conditions were finely selected for each main step of the expansion process. Expansion (pulling of the mandrel through the hole) was followed by the removal of the sleeve from the specimen, which was then reamed to the nominal functional diameter by removal of a calibrated set of elements in the hole edge. However, the removal of elements followed the initial mesh (before expansion) and thus did not correspond to actual "straight" drilling. This assumption was quite sufficient in this case, in view of the very small radial deformation along the hole surface. Finally, a relaxation step was included to reach the final equilibrium of the part (Fig. 11). This reaming simulation remains simplified considering the complexity of the phenomena involved in reality, where there are problems associated with machining, such as integrity of the

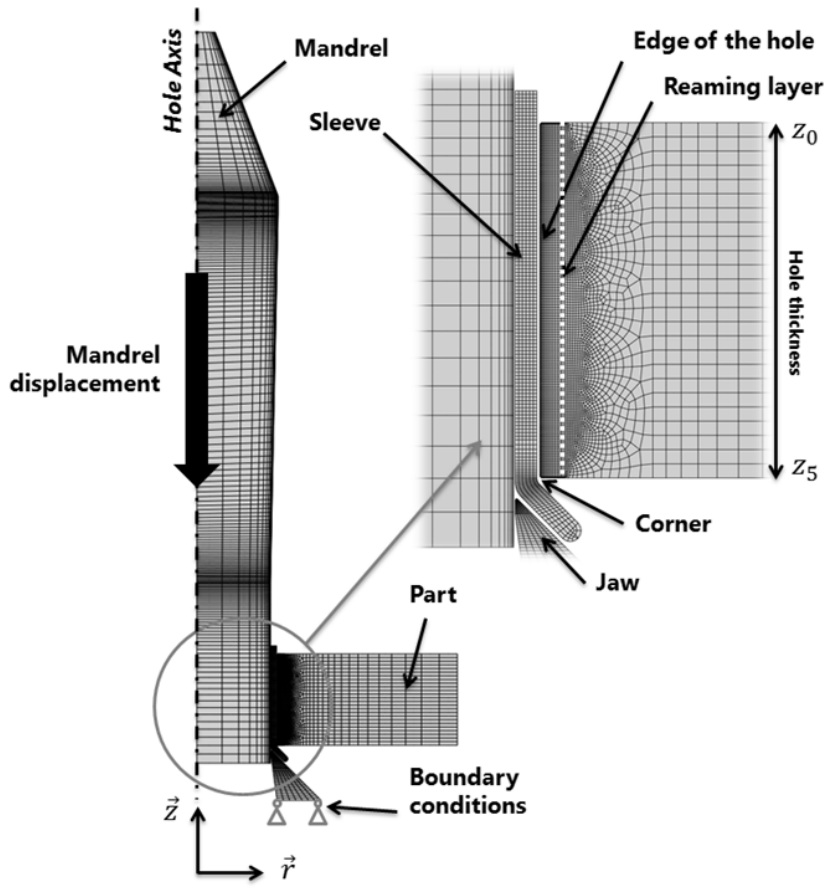

Fig. 10. Axisymmetric modelling of split sleeve expansion process.

surface generated, the displacement of the cutting tool or the heat generated and its possible influence on stress relaxation. Nevertheless, this strategy provided valuable information about the re-distribution of stress fields due to the removal of the contact zones between the expansion tooling and the part.

The geometry was chosen according to those used during the experimental fatigue study (Sect. 2.1), but only the $9.52 \mathrm{~mm}$ standard nominal diameters were studied. The model was thus composed of the net critical transverse sections of the tensile specimens presented in 


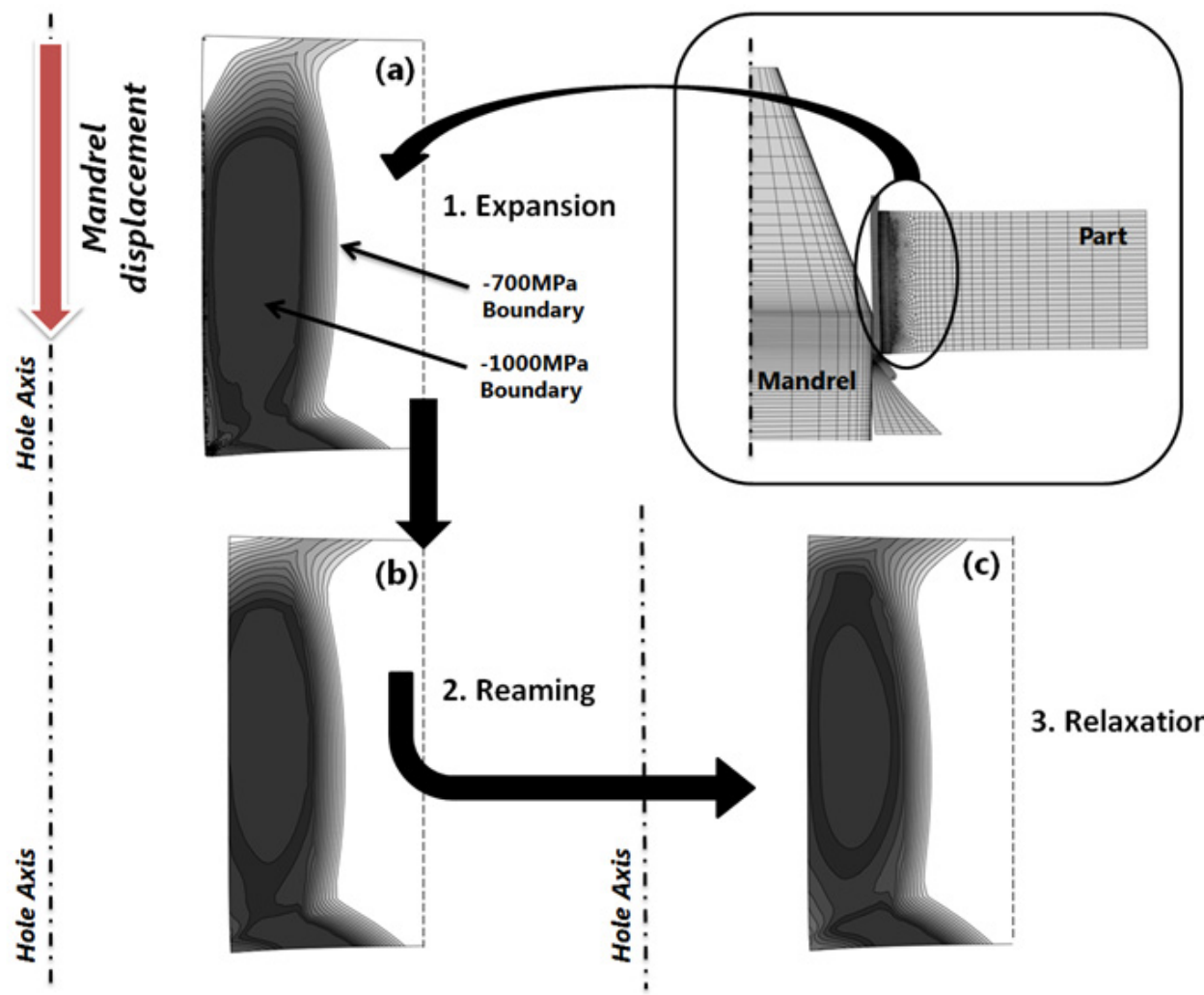

Fig. 11. Axisymmetric modelling of split sleeve expansion process: overview of residual circumferential stress fields, $>1000 \mathrm{MPa}$ (black), <700 MPa (grey). Reaming of the part (top) and relaxation/redistribution of residual fields (bottom).

Figure 8 and the expansion was performed by a tapered mandrel and a sleeve. Reaction to the axial loads generated during the expansion was obtained by axial locking of the jaw in order to be representative of the actual loading environment. Contact modelling between parts was selected with a penalty friction formulation and considering a hard contact. Friction coefficients ranged from 0.05 to 0.1 in the light of the materials in contact, the excellent surface integrity of the different parts and the high lubrication during the process. Meshing of the part and the sleeve was performed using quadrilateral 4-node bilinear elements including full integration (CAX8). Meshing of the mandrel and the jaw was performed using quadrilateral 4-node linear elements including reduced integration. The minimal size of the elements was $8 \times 10^{-3} \mathrm{~mm}$ close to the hole edge and reached $0.9 \mathrm{~mm}$ away from the edge. A parametric study determined the maximal size of the mesh that allowed good contact and no interference between the components at the corners (Fig. 10). A first assumption was made concerning the modelling of the sleeve. It was considered as an axisymmetric part whereas, in real conditions, the split allows slight opening of the sleeve, which may significantly reduce the hoop stresses within its section (Fig. 3). In practice, if a full sleeve is considered (i.e. without split), the sleeve is fully expanded and axial loads become excessive. The consequence is that the sleeve is caught by the mandrel, tends to slide on the jaw and exit from the hole surface. The modelling is then non-representative and includes excessive plastic flows. Convergence of the computation is then impossible. In order to overcome this problem, two assumptions were made. First, the sleeve was considered as a full axisymmetric part and the friction coefficient between the mandrel and the inner surface of the sleeve was reduced significantly. In this case, a 0.005 friction coefficient was chosen and the axial load transferred to the sleeve remained acceptable, simulating reasonable behaviour. We called this modelling choice "full sleeve". The other assumption was that the sleeve was an axisymmetric part with very low circumferential stiffness. This solution was intended to reduce axial load by easy expansion of the sleeve. The main advantage was to maintain a representative friction coefficient between the sleeve and the mandrel. We called this modelling choice "simulated split sleeve". In practice, this solution involved a friction coefficient of 0.025 and circumferential stiffness was divided by 1000 (arbitrary). A comparative study was performed concerning the influence of each assumption. Using these methodologies, computation times ranged between $1 \mathrm{~h}$ and $1.5 \mathrm{~h}$ on 4 CPUs.

Regarding the material model used, various solutions were compared. The goal was to determine which was most suitable to study the behaviour of the Ti-6Al-4V under complex triaxial stress when high plastic strains were involved. One of the main questions was how to account for the subsequent tension-compression of the material during expansion. On the one hand, this type of loading tends to involve kinematic issues and, on the other 


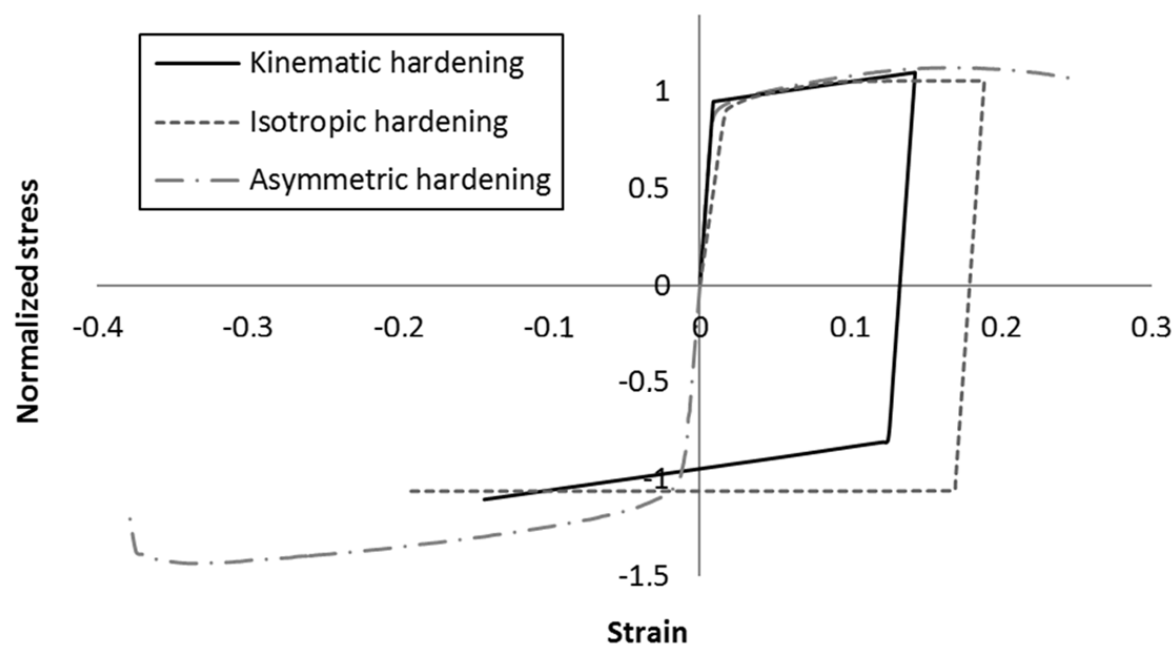

Fig. 12. Representation of material law: kinematic, isotropic and asymmetric hardening.

hand, it appears very important to be able to account for the different behaviours that may exist under tension and compression of the material. We have seen (Sect. 1.3) that $(\alpha+\beta)$ Titanium alloys may exhibit specificities regarding these two issues and a parametric study was performed regarding the hardening law to be considered. Its results were compared to experimental results in order to choose the right one for our study. Three material laws were tested (Fig. 12): first, a standard linear kinematic hardening law (one hardening slope) and, second, a pre-implemented anisotropic elasto-plastic law taking the tension-compression asymmetry of this alloy into account via the definition of two separate hardening laws, by linearization of the plastic behaviour between discrete values. All values were derived from characterization data obtained from quasi-static tests on Ti-6Al-4V $\alpha \beta$ specimens. These laws were finally compared with a multilinear isotropic hardening law. The material of the sleeve was martensitic steel with isotropic hardening, while the mandrel and jaws were simulated as elastic linear steel parts.

\subsection{Numerical results: identification of circumferential stress fields caused by cold expansion}

The results presented mainly concern the study of the circumferential stress, from the hole edge with regard to width of the plate at three different locations of the thickness of the specimens after split sleeve cold expansion. In order to sum up the observations, results taken from a simulation of "standard" expansion (4\%) and high expansion ratios (8\%) will be compared with our feasibility results to try to extract information regarding the associated fatigue performance. Because of its predominance in the onset and propagation of cracks in the hole section, only the circumferential stress will be analysed in this paper. Stresses are plotted over 3 paths corresponding to the face where the mandrel enters, the midsection of the specimen and the face where the mandrel exits. For the
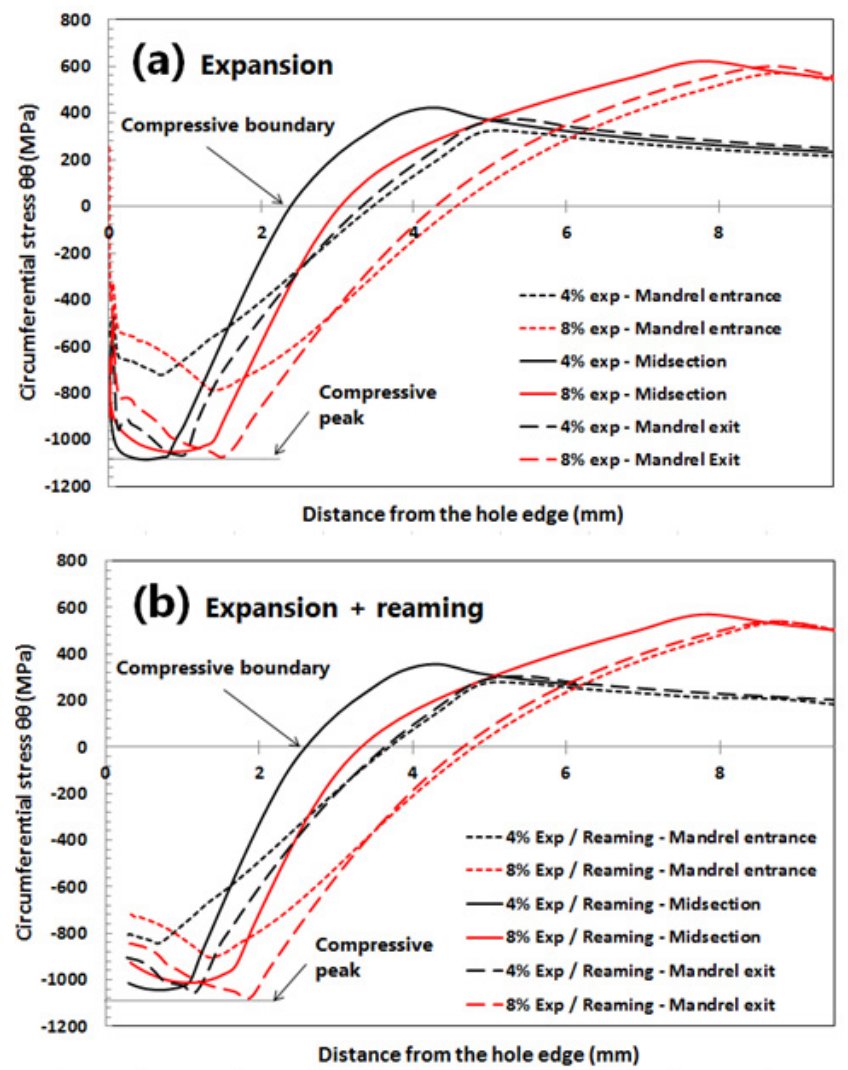

Fig. 13. Circumferential stress fields in entrance, mid and exit sections after expansion (a) and after expansion and reaming (b).

entrance and exit sections, paths are chosen at the first row of elements under the free surface to overcome some singularities existing near the hole surface.

First of all, Figure 13a allows the circumferential residual fields generated after cold expansion to be compared and Figure 13b shows these fields after cold expansion 


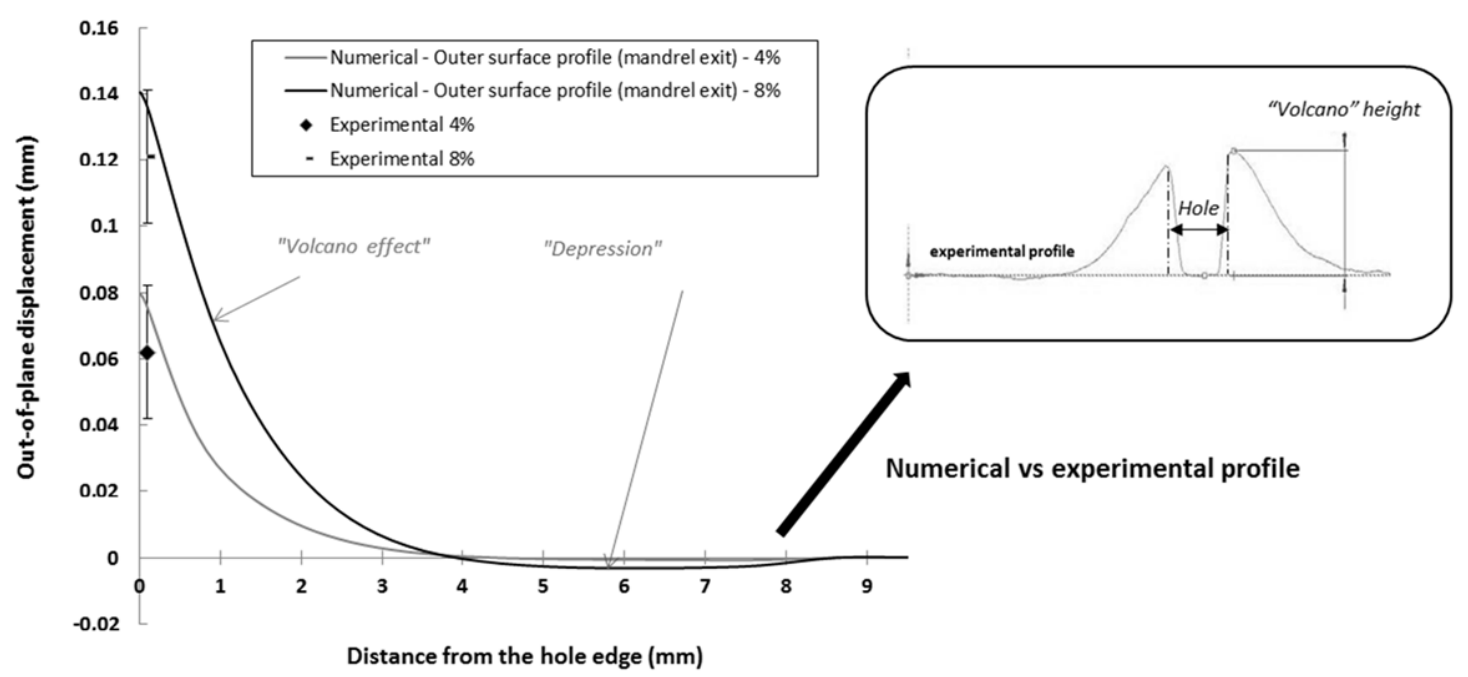

Fig. 14. Definition of the "Volcano Effect": Comparison between numerical and experimental results.

followed by a reaming to the functional diameter. These results were taken from simulation using kinematic hardening and the assumption of a full sleeve and reduction of the friction coefficient. We find the well-known phenomenon of the reduced compressive stresses at the entrance side of the mandrel. In our case, we were able to isolate and explain this phenomenon by the axial displacement of the material at the side where the mandrel entered and its "pushing away" preventing its radial expansion. At the mandrel entrance, the elements of the part are less constrained axially, much less than in the other sections (elements almost in plane stress state). On the other hand, it appears that this effect could be worsened because the sleeve is poorly maintained at the entrance of the hole and opens sharply although the mandrel penetrates into the hole. Figure 3 shows that the entrance face exhibits a larger area affected by the split of the sleeve (bulge). This means that this area concentrates most of the circumferential elongation, which may reduce the efficiency of the radial expansion of the overall material away from this area. Finally, we note that, in the mandrel entrance and exit sections, the compressive fields are more extensive than in the midsection (compressive boundary is $3.4 \mathrm{~mm}$ instead of $2.4 \mathrm{~mm}$ ).

In Figure 13a, we can see that high expansion ratios result in an increase in the extent of the compressive fields at the hole edge. In the mandrel entrance and exit sections, the compressive boundary is pushed back by $1 \mathrm{~mm}$ to $1.5 \mathrm{~mm}$. In the median section, this boundary is pushed back $0.7 \mathrm{~mm}$. Moreover, it is interesting to note that increasing the expansion ratio does not lead to greater intensities of the compressive peaks. Thus, for both $4 \%$ and $8 \%$, there is a compressive peak close to $-1075 \mathrm{MPa}$ at the mandrel exit in the midsection. At the entrance, the peak is slightly more compressive $(-70 \mathrm{MPa})$ for an expansion ratio of $8 \%$. Thus, only the width of the compressive peak becomes greater as the ratio increases. Furthermore, in the hole edge, the stress is less compressive, with a ratio of $8 \%$. It should also be noted that significant tensile stresses are generated away from the hole in order to achieve static equilibrium of the section. These stresses are doubled for an $8 \%$ expansion and may lead to crack initiation.

A characteristic of the split sleeve process is the generation of an out-of-plane residual deformation in the perimeter of the hole. This effect is usually called the "Volcano Effect" (Fig. 14). We know that this deformation can be very prejudicial for the behaviour of an assembly, the offset of material being responsible for significant fretting between the clamped plates [28]. It is, therefore, a phenomenon we want to minimize. The volcano was observed using our modelling strategy and a significant axial residual displacement was measured for $4 \%(0.079 \mathrm{~mm})$ and $8 \%(0.14 \mathrm{~mm})$. Experimentally, we measured these volcanoes with a profile measurement machine (Fig. 14). We measured $0.067 \mathrm{~mm}$ for $4 \%$ expansion and $0.121 \mathrm{~mm}$ for $8 \%$. However, this method leads to uncertainty of the order of $\pm 0.02 \mathrm{~mm}$, which comes primarily from the point and reference of the measure. Moreover, in Figure 15, the classical "inverted barrel" shape of the hole section can be observed, a consequence of the axial loading occurring due to the mandrel displacement. This deformation is thus amplified for high expansion ratios.

The influence of reaming was then evaluated (Fig. 13b). Reaming was simulated by deleting several rows of elements close the surface of the hole (the reaming layer is shown in Fig. 10). Then a static step of relaxation was performed so that the part was in static equilibrium. In general, the deletion of the reaming layer induced disappearance of the zero circumferential stress at the edge of the hole. We note that the reaming induced homogenization of the stress along the section. Stress peak intensities were closer at the edge of the hole, especially at the mandrel entrance position, where the stress became more compressive (about $-150 \mathrm{MPa}$ ). The volcano measured $0.068 \mathrm{~mm}$ for $4 \%$ expansion and $0.12 \mathrm{~mm}$ for $8 \%$ expansion. It was, however, noticed that the reaming induced a significant relaxation of the axial compressive residual 


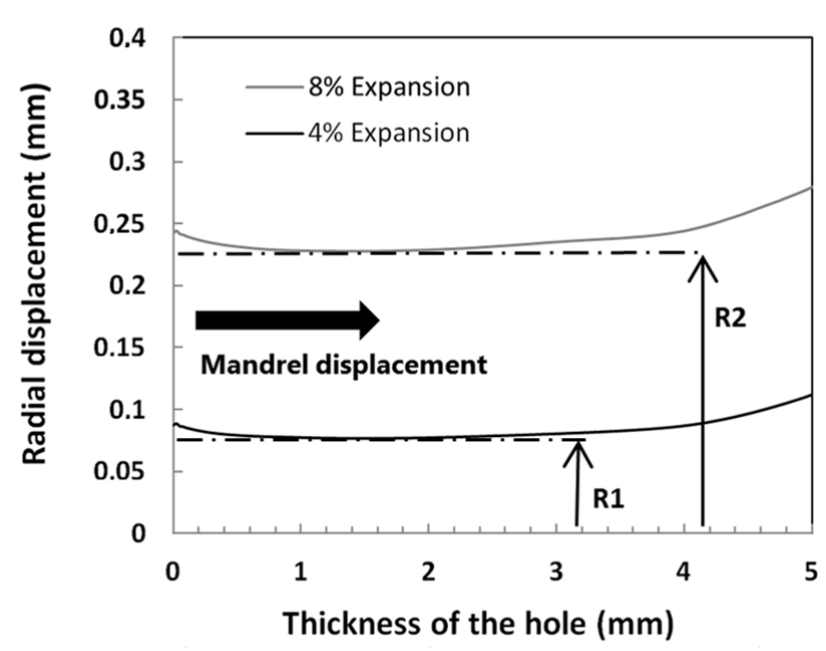

Fig. 15. Residual profile (radial deformation) of the hole along its thickness after cold expansion.

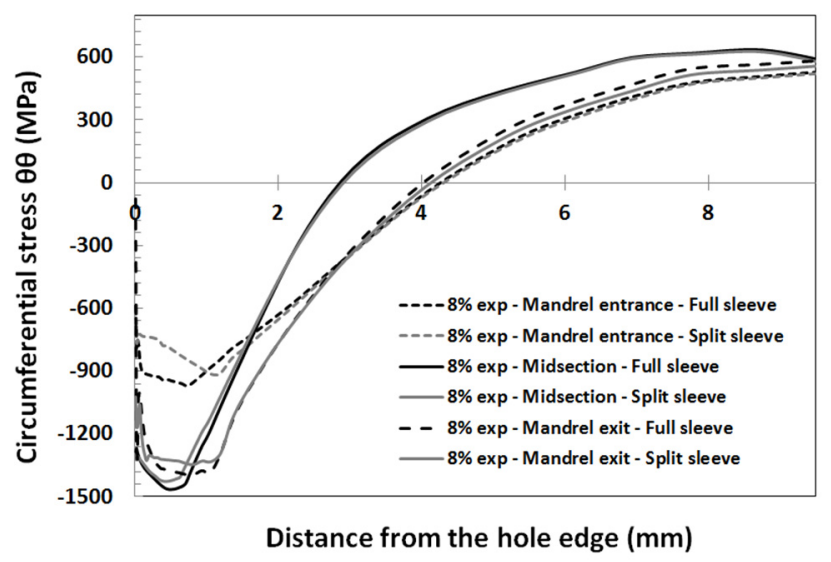

Fig. 16. Influence of sleeve modelling strategy.

stresses generated during the displacement of the mandrel (existing mainly in the deformed reaming layer). Numerically, this relaxation tended to increase the volcano, which is why values remained significant after reaming of the specimens. Specimens used for the experimental study were chamfered on both sides of the hole $\left(0.4 \times 45^{\circ}\right)$. The chamfer significantly reduced the volcano effect at the entrance and exit of the mandrel. Numerically, with an equivalent chamfer, the volcanoes measured were $0.25 \mathrm{~mm}$ for $4 \%$ expansion and $0.6 \mathrm{~mm}$ for $8 \%$ expansion.

A further simulation validated the assumptions chosen for the simulation of the process. First of all, the influence of the sleeve modelling strategy was considered. The results shown in Figure 16 are taken from simulations using asymmetric hardening. We can see that the sleeve modelling strategy has little influence on the associated residual fields after expansion and both strategies give nearly identical stress fields, except at the entrance face where the use of a "simulated split sleeve" induces a slight decrease in stress intensity. However, visual deformation of the sleeve tends to validate the assumption of a "full

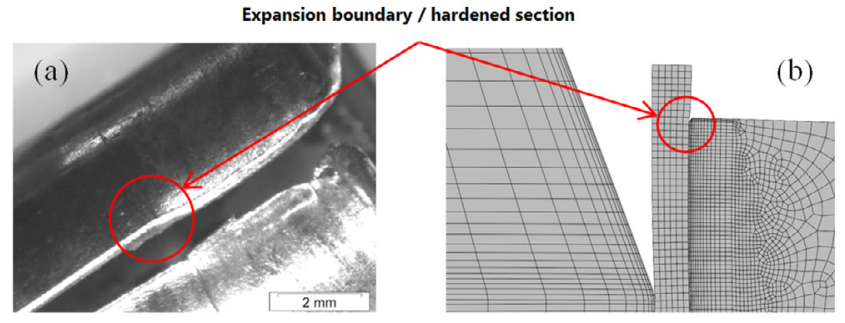

Fig. 17. Identification of the expansion boundary in the sleeve section: experimental (a) - Numerical (b) after high expansion ratio.

sleeve" and low friction coefficient between the mandrel and the sleeve. Significant hardening of the real sleeve during expansion was well simulated using this strategy (Fig. 17).

\subsection{Influence of the hardening law on residual fields and experimental correlation}

The next results presented allow the residual field and deformation generated during cold expansion to be compared for various hardening laws. As described in Section 3.1, the laws considered for the material included kinematic, isotropic and asymmetric hardening. The results show that, for standard and high expansion ratios, the choice of a hardening law is very important and has considerable influence on residual stress fields. Figures $18 \mathrm{a}-18 \mathrm{c}$ plot the residual fields generated after $8 \%$ of cold expansion considering the three hardening laws. It appears that, in this range of expansion ratios, the value of the compressive peak is highly dependent on the law chosen for the material. Thus, if we use a law sensitive to the decrease in yield strength after bidirectional hardening, we can see a decrease of the intensity of the compressive peak. In contrast, the use of an anisotropic hardening law, which considers the high compressive properties of the Ti- $6 \mathrm{Al}-4 \mathrm{~V}$, shows an increase of the intensity of the compressive peak. However, we can see that there is no change in the general trends relating to the residual fields generated and, in particular, there is no change in the compressive boundaries in the entrance, exit and mid sections. As long as we find means of measuring the value of the compressive peak precisely, we have the ability to refine these elasto-plastic laws in order to obtain a reliable model for predicting the stress distribution in the expanded section of hard alloys.

In order to validate the choice of a hardening law for our study, the numerical and experimental dimensional results obtained after expansion were compared. First of all, the values obtained by measuring the volcano height were compared. As seen in Section 3.2, they were very close numerically and experimentally: whatever the material law used, the height was within $\pm 0.02 \mathrm{~mm}$ of the experimental value measured. However, it was not possible to compare and justify one material law with respect to another because the scatter observed experimentally was also in the $\pm 0.02 \mathrm{~mm}$ range. For this reason, 


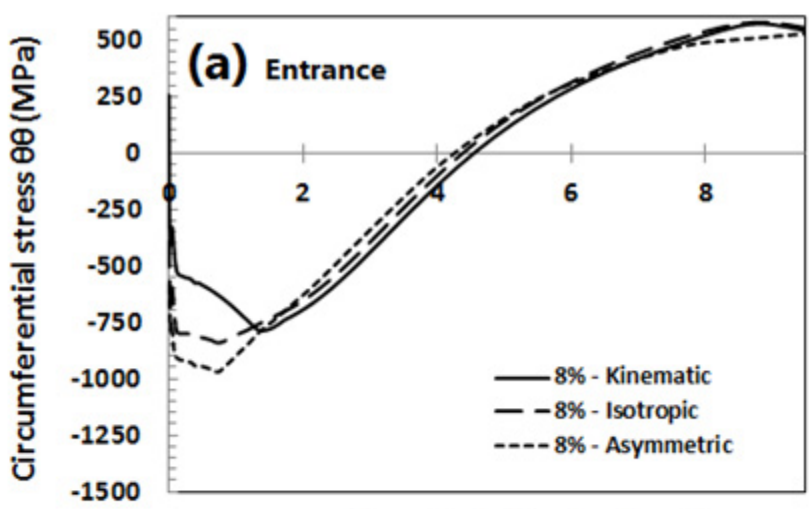

Distance from the hole edge $(\mathrm{mm})$

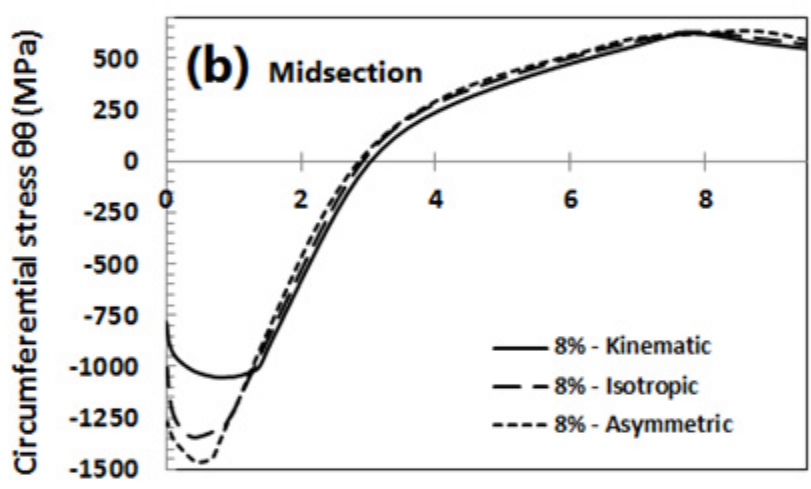

Distance from the hole edge $(\mathrm{mm})$

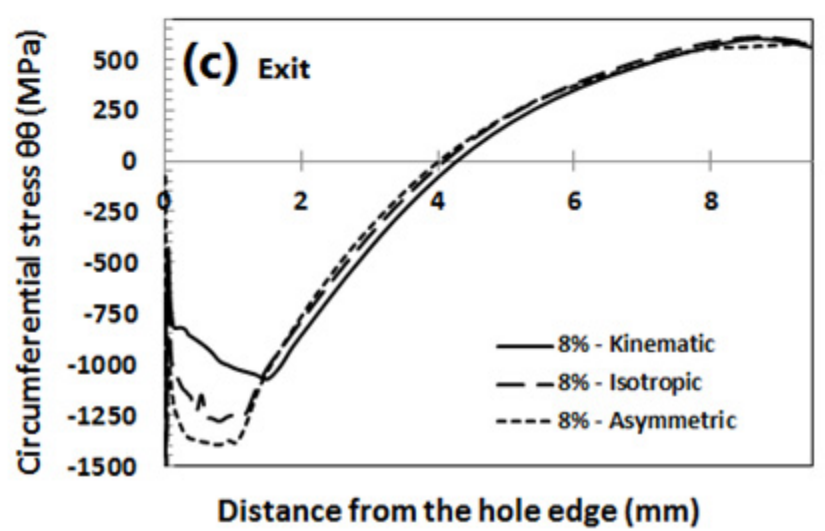

Fig. 18. Evolution of circumferential residual fields in entrance section (a), midsection (b) and exit section (c) according to the diverse hardening laws.

we chose to compare the values obtained from the measured residual expansion ratio (before reaming) as these very specific results, presented in Section 2.1, showed very little spread and could be compared to the numerical dimension measured as shown in Figure 15 (dimensions R1 and R2). Thus, a very good correlation of these variables can be observed in Figures 19a and 19b for two material laws. In fact, our model was able to predict the residual expansion, which is highly related to the hardening model, with great accuracy. Thus, using the isotropic and kinematic hardening laws, we could predict the residual expansion with an accuracy within the experimental dispersion. Moreover, the values obtained were extremely close to the average values of the experimental tests. Only the anisotropic hardening law was unable to predict the value of the residual expansion successfully. Thus, by comparing the results for the two expansion levels tested, we obtained a percentage of error lower than $3.8 \%$ for a $4 \%$ expansion. At $8 \%$ expansion, this error was lower than $2 \%$.

\section{Conclusion and discussion}

The present study focused on the assessment and understanding of the cold expansion process for holes in hard alloys. These results provide the first information regarding the application of the expansion process to hard alloys and should help to define an approach for understanding which processes and methodologies are suitable for the efficient expansion of hard metals. The main observations and conclusions are:

- The split sleeve cold expansion tooling is functional on hard alloy holes. Moreover, titanium alloys, nickelbased alloys and martensitic steels can sustain high expansion ratios (up to 8\%). In this expansion range, residual expansion ratio grows linearly with the theoretical expansion ratio. Each alloy exhibits highly specific behaviour, e.g. large springback. On titanium and nickel-based alloys the absence of scale effect could be observed for $6.35 \mathrm{~mm}$ and $9.52 \mathrm{~mm}$ diameter holes.

- Fatigue tests on open-hole specimens showed that improved fatigue performance was observed using the split sleeve cold expansion process. Two expansion ratios were considered, a "standard" expansion ratio and a high expansion ratio. On two hole diameters, greater gains were observed with high expansion ratios. Particularly, gains are even more notable for low number of loading cycles ( $<200000$ cycles). Different failure modes were observed. The large majority of specimens showed failure in the net section. However, we also observed "offset" failure modes.

- Axisymmetric FE modelling was developed in order to simulate the different steps of the split sleeve process and explain some of the experimental results. The aim was to understand what phenomena were involved after the application of a high expansion ratio in comparison with a "standard" ratio.

- The circumferential stress was analysed on a "standard" expansion ratio of $4 \%$ and a high expansion ratio of $8 \%$. Results show that intense, extensive compressive fields were generated after cold expansion. For both "standard" and high ratios, we found the wellknown phenomenon of lower compressive stresses at the entrance side of the mandrel. In our case, an explanation of this phenomenon could be proposed. In contrast, we noted that the compressive fields were more extensive in the mandrel entrance and exit sections than in midsection. 

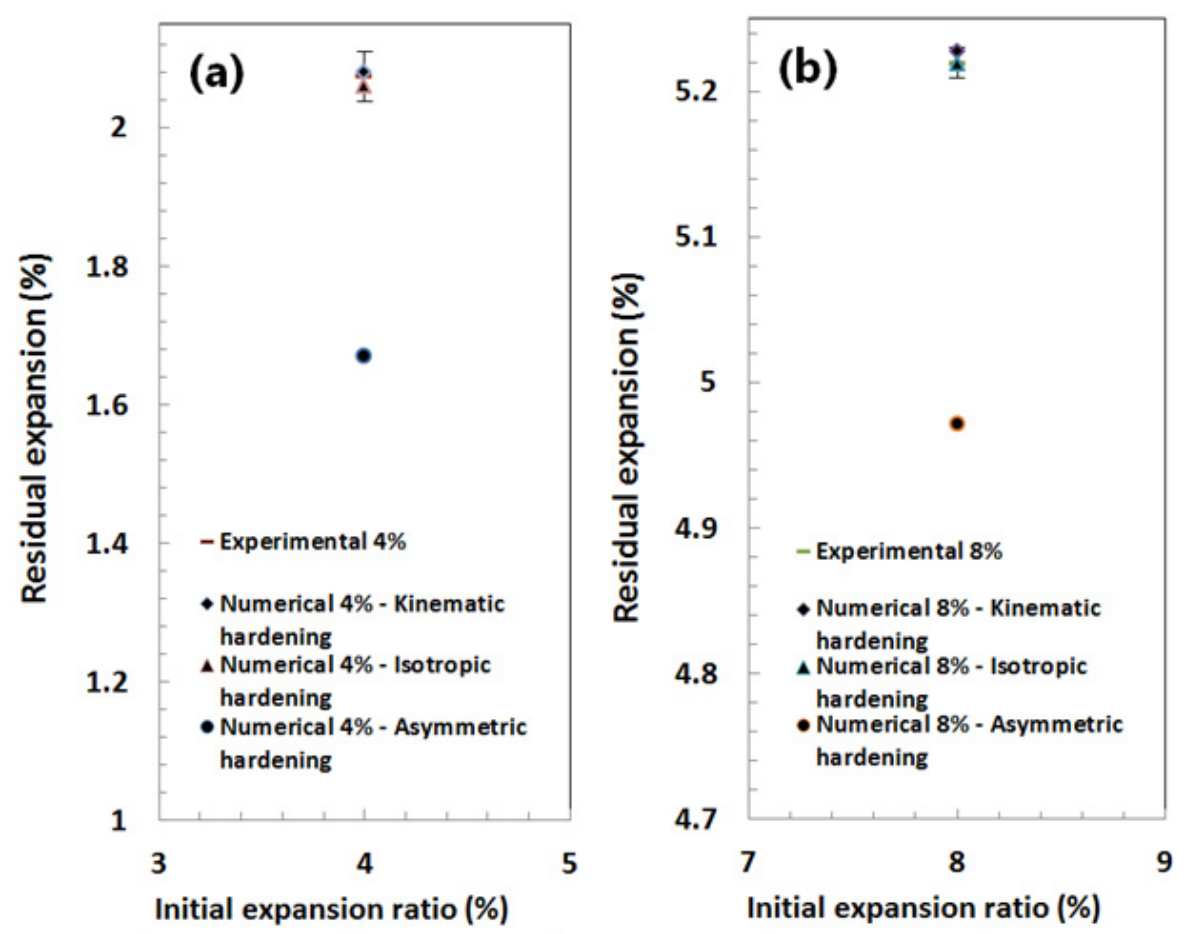

Fig. 19. Analysis of the residual expansion ratio: correlation between experimental and numerical results for $4 \%$ expansion ratio (a) and $8 \%$ expansion ratio (b).

- Simulations showed that high expansion ratios resulted in an increase of the extent of the compressive fields at the hole edge. Over the thickness of the hole, the compressive boundaries were pushed back. Moreover, it is interesting to note that increasing the expansion ratio did not achieve greater intensities of the compressive peaks located close to the hole edge but it must be observed that significant tensile stresses were generated away from the hole in order to achieve static equilibrium of the section. These stresses were doubled for the high expansion ratio and could involve crack initiation. The results also show that high expansion ratios tended to increase the volcano effect at the exit of the mandrel and the "inverted barrel" shape of the hole.

- The influence of reaming the hole after cold expansion was evaluated. In general, the deletion of the reaming layer led to the disappearance of the zero circumferential stress at the edge of the hole. Moreover, the reaming caused homogenization of the circumferential stress along the section.

- Three different hardening (kinematic, isotropic and asymmetric) laws were considered in order to faithfully simulate several specific behaviours of $(\alpha+\beta)$ titanium alloys. The choice of a hardening law has a great impact on the value of the compressive peak, but not on the location of the compressive boundary within the part.

- Experimental and numerical comparisons were made. Good agreement of the results was observed when analysing the volcano height. The model predicted the measured residual expansion ratio for "standard" and high expansion ratios very precisely $(<3.8 \%)$. Thus, this model is able to provide high accuracy results as long as it is possible to determine the intensity of the compressive peak experiments for calibration. These results could thus be implemented in such models for fatigue life or crack propagation prediction around cold expanded holes.

This study has especially helped to isolate the role and the importance of a high expansion ratio on the fatigue strength of hard alloys. Seeing the numerical results obtained, it is possible to state that the improvement of the fatigue strength observed experimentally with high expansion ratios is presumably due to the extension of the compressive area (compressive boundary within the section). Presumably, a wider compressive area may have two actions on the fatigue life. We can first assume that this larger area is responsible for a stronger deviation of the stress flows at the hole edge during subsequent external loading. Thus, this deviation induces very efficient "isolation" of the critical area. Moreover, we can assume that this larger compressive area is responsible for a significant slowdown of the growth rate of fatigue cracks (significant decrease in Kic at the crack tip). So, if this area is more extensive, its influence on the large propagating cracks is greater. In fact, the improvement observed in fatigue performance with high expansion ratios is probably a consequence of both actions. 


\section{References}

[1] E.O. Ezugwu, Key improvements in the machining of difficult-to-cut aerospace superalloys, Int. J. Machine Tools Manuf. 45 (2005) 1353-1367

[2] R.E. Peterson, Stress concentration design factors, Wiley, New York, 1953

[3] J. Liu, Z.F. Yue, Y.S. Liu, Surface finish of open holes on fatigue life, Theoret. Appl. Fracture Mech. 47 (2007) $35-45$

[4] G.I. Taylor, The Mechanism of Plastic Deformation of Crystals. Part I, Proceedings of the Royal Society of London. Series A, Containing Papers of a Mathematical and Physical Character, 1934, No. 362: 145, p. 855

[5] M. Paredes, R. Canivenc, M. Sartor, Tolerance optimization by modification of Taguchi's robust design approach and considering performance levels: Application to the design of a cold-expanded bushing, Proceedings of the Institution of Mechanical Engineers, Part G: Journal of Aerospace Engineering, 2013

[6] T. Benhaddou, P. Stephan, A. Daidié, C. Chirol, J-B. Tuery, Effect of Axial Preload on Double-Lap Bolted Joints: Numerical Study, Nantes: Proceedings of ASME 2012, 11th Biennial Conference On Engineering Systems Design And Analysis, 2012

[7] M. Paredes, N. Naoufel, M. Sartor, Study of an interference fit fastener assembly by finite element modelling, analysis and experiment, Int. J. Interactive Design Manufact. 6 (2012) 171-177

[8] L.F. Reid, Advanced structural fastening and jointing methods utilizing synergistic cold expansion and high interference fit methodology, European Conference for Aerospace Sciences (EUCASS)

[9] A.C. Rufin, Extending the fatigue life of aircraft engine components by hole cold expansion technology, J. Eng. Gas Turbines Power 115 (1993) 165-171

[10] X. Zhang, Z.Wang, Fatigue life improvement in fatigue aged fastener holes using the cold expansion technique, Int. J. Fatigue 25 (2003) 1249-1257

[11] A.T. Ozdemir, R. Hermann, Effect of expansion technique and plate thickness on near-hole residual stresses and fatigue life of cold expanded holes, J. Mater. Sci. 34 (1999) 1243-1252

[12] L. Phillips Joseph, Technical Sleeve coldworking of fastener holes, Air Force Materials Laboratory (SAE Paper no. 73095), AFML-TR-74-10, 1974

[13] D.L. Rich, L.F. Impellizzeri, Fatigue Analysis of Coldworked and Interference Fit Fastener Holes/Cyclic StressStrain and Plastic Deformation Aspects of Fatigue Crack Growth, ASTM STP (ASTM STP) 637 (1977) 153-175

[14] G.T. Sha, B.A. Cowles, R.L. Fowler, Fatigue Life of a Coldworked hole, In Emerging technologies in aerospace structures, design structural dynamics and materials, by Jack R. Vinson. American Society of Mechanical Engineers. Aerospace Division, 1980, pp. 125-140
[15] W.Z. Yan, X.S. Wang, H.S. Gao, Z.F. Yue, Effect of split sleeve cold expansion on cracking behaviors of titanium alloy TC4 holes, Eng. Fract. Mech. 88 (2012) 79-89

[16] J. Liu, H. Wu, J. Yang, Z. Yue, Effect of edge distance ratio on residual stresses induced by cold expansion and fatigue life of TC4 plates, Eng. Fract. Mech. 109 (2013) 130-137

[17] B.D. Flinn, M.E. Wiegman, M. Sigelmann, E. Easterbrook , A. Mines, StressWave fatigue life improvement of $6 \mathrm{Al}-4 \mathrm{~V}$ titanium for medical implants, Proceedings of the materials and processes for medical devices conference, 2003

[18] StressWave, Fatigue testing of 6Al-4V titanium, 2001

[19] E.-L. Odenbergera, M. Oldenburgb, P. Thilderkvista, T. Stoehrc, J. Lechlerc, M. Merklein, Tool development based on modelling and simulation of hot sheet metal forming of Ti-6Al-4V titanium alloy, J. Mater. Process. Technol. 211 (2011) 1324-1335

[20] J.R. Mayeur, D.L. McDowell, A three-dimensional crystal plasticity model for duplex Ti-6Al-4V, Int. J. Plasticity 23 (2007) 1457-1485

[21] J.A. Medina Perilla, J.Gil. Sevillano, Two-dimensional sections of the yield locus of a Ti-6\%Al- $4 \% \mathrm{~V}$ alloy with a strong transverse-type crystallographic a-texture, Materials Science and Engineering, 1995

[22] S. Khan Akhtar, Yu. Shaojuan, Deformation induced anisotropic responses of Ti-6Al-4V alloy Part I: Experiments, Int. J. Plasticity, 2012

[23] E.W. Collings, Applied superconductivity, metallurgy, and physics of titanium alloys, Plenum Press, New York, 1986

[24] T.N. Charkherlou, J. Vogwell, The effect of cold expansion on improving the fatigue life of fastener holes, J. Eng. Failure Anal. 10 (2003) 13-24

[25] V. Nigrelli, S. Pasta, Finite-element simulation of residual stress induced by split-sleeve cold-expansion process of holes, J. Materi. Process. Technol. 205 (2008) 290-296

[26] E.T. Easterbrook, M.A. Landy, Evaluation of the StressWave cold working (SWCW) process on high strength aluminium alloys for aerospace, AIR FORCE RESEARCH LABORATORY AFRL-RX-WP-TR-20094027, 2009

[27] T.N. Charkherlou, J. Vogwell, A novel method of cold expansion which creates near-uniform compressive tangential residual stress around a fastener hole, Fatigue Fract. Eng. Mater. Struct. 27 (2004) 343-351

[28] L. Boni, A. Lanciotti, C. Polese, Some contraindications of hole expansion in riveted joints, Eng. Failure Anal. 46 (2014) 140-156

[29] M.E. Karabin, F. Barlat, R.W. Schultz, Numerical and experimental study of the cold expansion process in 7085 plate using a modified split sleeve, J. Mater. Process. Technol. 189 (2007) 45-57 